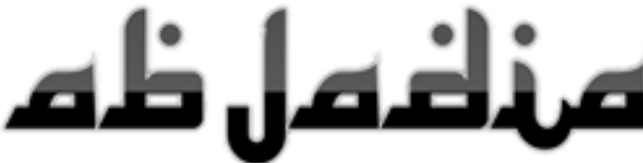

International Journal of Education

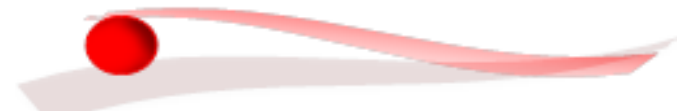

Pi] Publisher

Fakultas Ilmu Tarbiyah dan Keguruan Universitas Islam Negeri (UIN] Mualana Malik Ibrahim Malang Indonesia

2443-0587 - ISSN Online

2528-3979 - ISSN Print

霄 History Article

t) Received : 10-03-2017

(1⿰⿸尸口⿱⿱亠䒑十纟) Reviced : 29-03-2017

目 Accepted : 16-06-2017

\section{doi.org/ 10.18860/abj.v2i1.5309}

http://ejournal.uin-malang.ac.id/index.php/ abjadia/article/view/5309

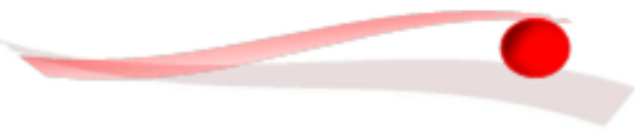

Zenib Moharned M. Alfegeh

I. Libya

University of Zawia - Libya

\section{Corresponding Author}

(C) 081554733331

zomd2015@gmail.com

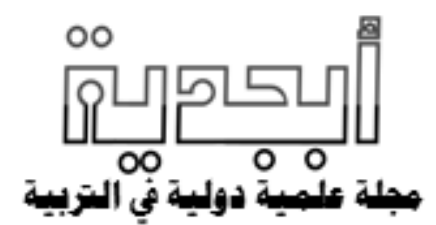

Zenib Mohamed M. Alfegeh

Libya

أهمية تكنولوجيا المعلومات في دعم التطوير التنظيمي

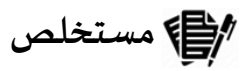

يشير مصطلح تكنولوجيا المعلومات إلى مجموعة المعرفة العلمية

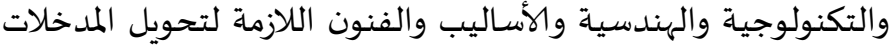

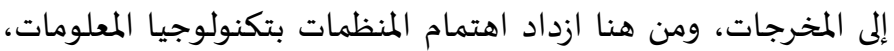

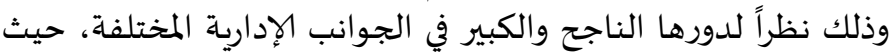

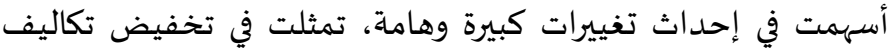

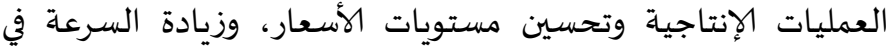

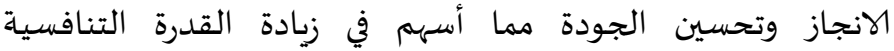

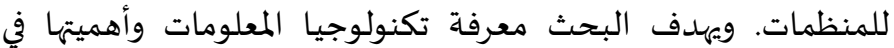

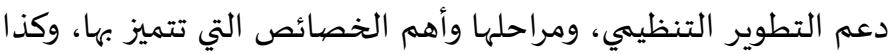

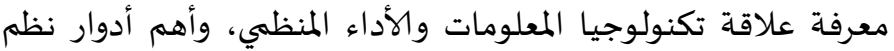

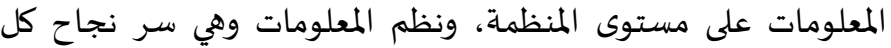

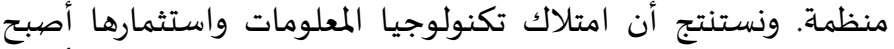

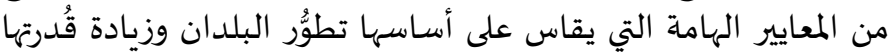

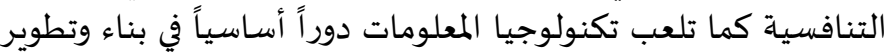

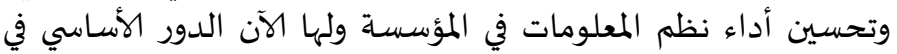

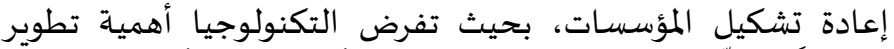
نموذجاً إداريًا يتميز بالانفتاح والمرونة والتكيُّف مع التطوُّرات التقنية. تكنولوجيا المعلومات ، التعليم، الإدارة

\section{䝿”" Abstract}

Information technology refers to the range of scientific, technological and engineering knowledge, methods and arts needed to transform inputs into outputs. Hence, the organizations' interest in information technology has increased due to their successful and significant role in the various administrative aspects, contributing to significant and significant changes, Improved price levels, increased speed of delivery and improved quality, which contributed to increased competitiveness of organizations. The research aims at identifying the information technology and its importance in supporting organizational development, its stages and the main characteristics that characterize it, as well as knowledge of the relationship of information technology and organizational performance, the important roles of information systems at the organizational level, and information systems. It is concluded that the acquisition and investment of information technology has become an important criterion on which the development and competitiveness of countries are measured. Information technology plays an essential role in building, developing and improving the performance of information systems in the enterprise. Characterized by openness, flexibility and adaptation to technological developments.

\section{Information Technology, Education, Management}




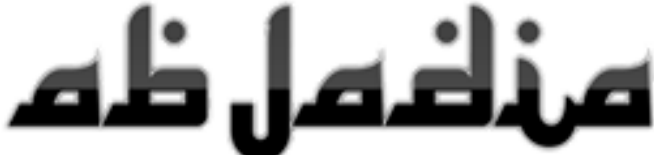
International Journal of Education

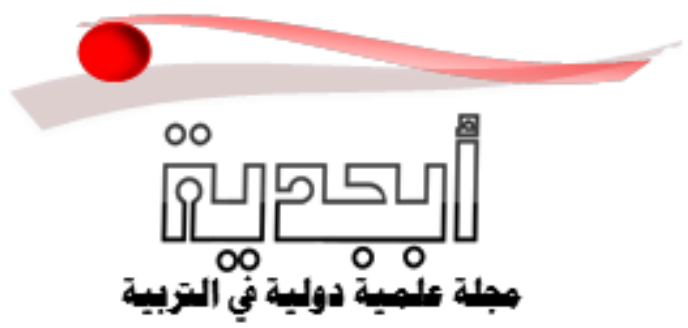

\section{䨢" Abstrak}

Istilah Teknologi Informasi mengacu pada pengetahuan ilmiah, teknologi, teknik, metode dan seni untuk mengubah input menjadi output. Karena itu, minat organisasi (lembaga) terhadap teknologi informasi terus meningkat karena berhasil dalam aspek administratif, berkontribusi pada perubahan, nilai jual, meningkatkan kecepatan, kualitas pengiriman, dan daya saing. Tujuan penelitian ini memperkenalkan teknologi informasi dan urgensinya dalam mendukung pengembangan organisasi, menampilkan tahapan teknologi informasi dan karakteristik, hubungan teknologi informasi dan kinerja organisasi, dan peran penting sistem informasi. Dapat disimpulkan bahwa perolehan dan investasi teknologi informasi menjadi penting karena perkembangan negara dan daya saingnya bisa diukur. Teknologi informasi berperan penting membangun, mengembangkan dan meningkatkan kinerja sistem informasi di lembaga pendidikan dan memiliki peran restrukturisasi institusi. Secara administratif, teknologi informasi dicirikan oleh keterbukaan, fleksibilitas dan kemampuan beradaptasi terhadap perkembangan teknis

?. Teknologi Informasi, Pendidikan, Manajemen

http:// $\quad$ http://ejournal.uin-malang.ac.id/index.php/abjadia/article/view/5309

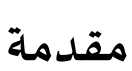

أدى التطور التكنولوجي السريع والحاجة المتزايدة إلى السرعة في الانجاز ورغبة المواطنين في

الحصول على خدمات عديدة وبصورة أكثر تطوراً وبدقة متناهية مع قصور الإدارة التقليدياة للاستجابة لتلك الرغبات إلى ضرورة إعادة النظر في نظم وأساليب إدارة الخدمات، ومن هنا ازداد اهتمام المنظمات بتكنولوجيا المعلومات، وذلك نظرا لدورها الناجح والكبير في الجوانب الإدارية المختلفة، حيث أسهمت في إحداث تغييرات كبيرة وهامة، تمثلت في تخفيض تكاليف العمليات الإنتاجية وتحسين مستويات الأسعار، وزيادة السرعة في الانجاز وتحسين الجودة مما أسهم في زيادة القدرة التنافسية لهذه المنظمات، وتحقيق أهدافها في البقاء والنمو والتوسع في أداء أعمالها، بالاعتماد على ما توفره هذه التكنولوجيا من وسائل وأدوات مناسبة.

ولقد تعددت مفاهيم تكنولوجيا المعلومات وفقا لآراء الكتاب والباحثين في إعطائهم مفهوما واحدا شاملا ولعل مرد ذلك يرجع لاختلاف مرجعياتهم العلمية ومنطلقاتهم الفكرية فمنهم من عرفها بأهها: مجموعة المعرفة العلمية والتكنولوجية والهندسية والأساليب والفنون اللازمة لتحوبل المدخلات إلى

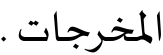


أهداف البحث هي: 1) التعريف بتكنولوجيا المعلومات وأهميتها في دعم التطوير التنظيمي؛ 2) معرفة مراحل تكنولوجيا المعلومات وأهم الخصائص التي تتميز بها؛ 3) معرفة علاقة تكنولوجيا المعلومات والأداء المنظمي؛ 4) معرفة أهم أدوار نظم المعلومات على مستوى المنظمة؛ 5) معرفة أن نظم المعلومات سر نجاح كل منظمة.

\section{[0] منهج}

منهج هذا البحث كيفي أو نوعي. وعُرض البحث وصفيا لمعرفة حقيقة تكنولوجيا المعلومات، وأهميته، ومراحله، وخصائصها، وعلاقتاه بالأداء المنظمي، وكذا أدوار نظم المعلومات على مستوى المنظمة. أما أسلوب جمع البيات الذي أجرته الباحثة هو الدراسـة المكتبية حيث طلبت الباحثة جميع ما يتعلق بتكنولوجيا المعلومات. وإذا اجتمعت البيانات قامت الباحثة بقراءتها ومطالعتها وتصنيفها وتحليلها حسب الإطار النظري المحتوي على مباحث تكنولوجيا المعلومات.

\section{نتائج مفهوم تكنولوجيا المعلومات}

تعددت مفاهيم تكنولوجيا المعلومات وفقا لآراء المهتمين (Daft 2000)، بأها سلاح استراتيجي يمكن أن يساعد في بناء وتعزيز قدرات المنظمة الاستراتيجية من خلال توفير أفضل البيانات والمعلومات داخلها وخارجها وبما يوطد علاقة المنظمة بالمجهزين والزبائن والمنظمات الأخرى. كما أن تكنولوجيا المعلومات هي الجانب التكنولوجي من نظام المعلومات والذي يمثل المكونات المادية، البرمجيات، قواعد البيانات، الشبكات والوسائط الاخرى. وعبر عنها كونها مجموعة من الافراد، البيانات، الاجراءات، المكونات المادية Slack, Nigel \& Chambens, S. \& Harland , C. \& ) والبرمجية التي تعمل من أجل تحقيق اهداف المنظمة .(Harrision, A. \& Johanston 1998

كما أهها تعني الأدوات والتقنيات والنظم التي يمكن استخدامها للحصول على المعلومات والبيانات ومعالجتها وخزنها واسـترجاعها. اذ تتضهن هذه التقنيات الحاسوب بأنواعاه، اسـاليب الخزن والطبع والقراءة فضلا عن اساليب الاتلام والنقل والساتلايت وشبكات المحمول، والفاكس وأنظمة البرمجيات وتطبيقاتها. وتم تعريفها على انها المكونات المادية للحاسوب والبرمجيات، البيانات وتقنيات الخزن من 
حفظ وتوفير المعلومات والبيانات كموارد لتكنولوجيا معلومات المنظمة ) ر , Loudon , K and Loudon (2004

ان تكنولوجيا المعلومات تمثيل للجانب التكنولوجي لنظام المعلومات (حجيم et al. eم:). (كما تستخدم كبديل لها في احيان اخرى) فبينما اعتمدت مهمة معالجة البيانات وخزن المعلومات وتحديثها

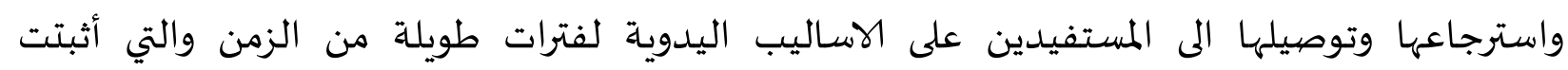

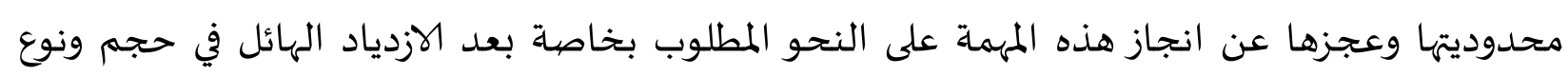
البيانات وبات الوضع يحتم ضرورة استخدام تكنولوجيا المعلومات الحديثة في تطبيقات نظام المعلومات.

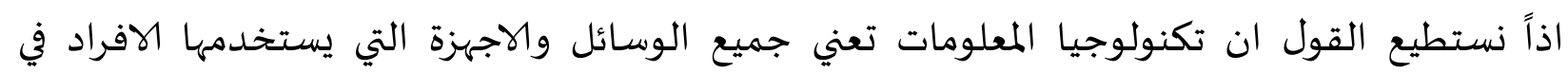

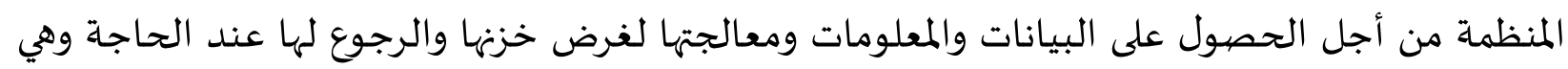

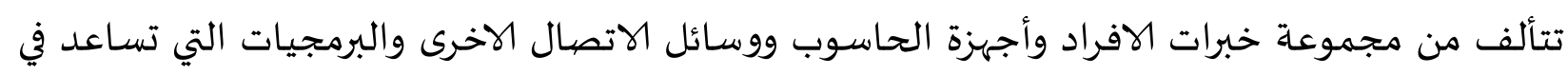

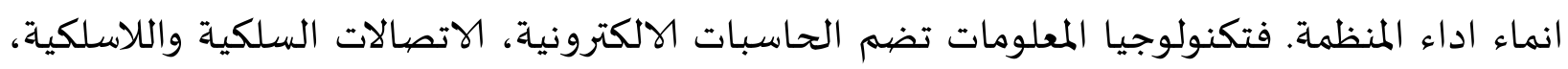
التكنولوجيا المسموعة والمرئية والطباعة والبرمجيات والخبرات والمهارات المتراكمة والمتاحة والوسائل

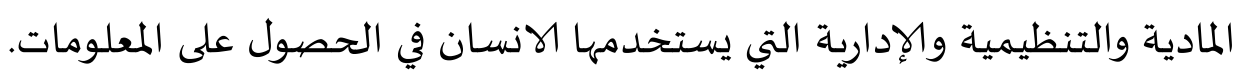
أهمية تكنولوجيا المعلومات

تبرز أهمية تكنولوجيا المعلومات من كونها تساعد المنظمات في الحصول على المعلومات المطلوبة

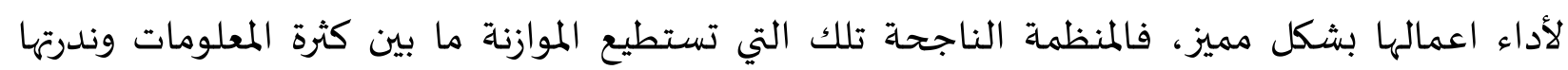
فتكنولوجيا المعلومات تساعد المنظمات في ايجاد فرص جديدة للعمل (Turban, Miclean, and (1999). وتزداد أهميتها من خلال توفيرها معلومات واسعة ودقيقة للمدراء مما يساعدهم في السيطرة على تنفيذ قراراتهم من مرؤوسيهم وهذا ربما يعود بهم الى المركزية من خلال الرقابة التي ستوفرها لهم أدوات تكنولوجيا المعلومات (صبري 2002).

إن أهمية تكنولوجيا المعلومات تبرز من خلال ما تمنحه للمنظمات من فرص سوقية جديدة لأهها

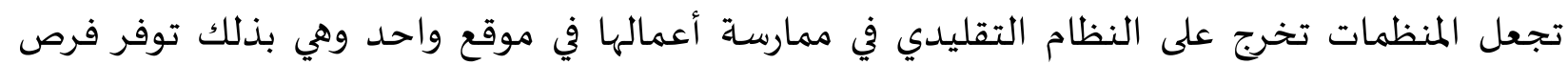
لإقامة شبكات أعمال بين أطراف متفرقة في شتى أنحاء العالم.

وتأسيسا "على ما تقدم فأن أهمية تكنولوجيا المعلومات تبرز من خلال ما توفره من اسهامات للعاملين والمديرين وذلك باطلاعهه على المعلومات، اذ ان المنظمات التي تكون في مجال المنافسة العالمياة 
لابد لها أن تمتلك عناصر ومقومات الميزة التنافسية المستقبلية والتي تعد تكنولوجيا المعلومات جزء هام

لقد مرت تكنولوجيا المعلومات بثلاثة مراحل أساسية، حيث اهتمت في بادئ الأمر بمعالجة البيانات، ثم انتقلت في المرحلة الثانية إلى معالجة المعلومات، وأخيرا أصبحت تركز على معالجة المعرفة. وهو ما يمكن توضيحها على النحو التالي (بركات 2005):

المرحلة الأولى: مرحلة معالجة البيانات: وبدأت هذه المرحلة مع منتصف الخمسينات من القرن العشرين، ولقد اتسمت بمحدودية الإمكانيات والتطبيقات التي تتيحها تكنولوجيا المعلومات، والتي اقتصرت على المعالجة الآلية للبيانات بهدف الحصول على المعلومات التي تلبي احتياجات المستفيدين في الوقت المناسب وبالتكلفة المناسبة. ومن أهم التطبيقات التي وفرتها تكنولوجيا المعلومات في هذا الصددد: برامج حسابات العملاء، برامج حسابات الموردين، برامج مراقبة المخزون، برامج المرتبات، نظم إدارة قواعد البيانات ونظم معالجة الكلمات والنشر المكتبي.

المرحلة الثانية: مرحلة معالجة المعلومات: بدأت هذه المرحلة خلال عقد الستينات

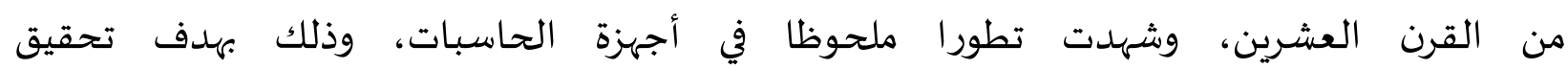
الترابط والتكامل بين المجالات الوظيفية المختلفة داخل المنظمة، وهو ما أدى إلى ظهوره عدد من نظم المعلومات الوظيفية، مثل نظم معلومات التسويق ونظم معلومات المهات الموارد البشرية.

المرحلة الثالثة: مرحلة معالجة المعرفة: وبدأت خلال عقد الثمانينات من القرن العشرين، وشهدت تطورا ملحوظا في البرمجيات وظهور تطبيقات الذكاء الاصطناعي والنظم الخبيرة، مما ساهم في تحسين القدرات والإمكانات التي تتيحها تكنولوجيا المعلومات، وزيادة قدرتها على محاكاة السلوك البشري. خصائص تكنولوجيا المعلومات تتمثل اهم خصائص تكنولوجيا المعلومات بالآتي (منير, ونعيمة, and بارك 2005): الأولى: تساهم تكنولوجيا المعلومات في تحقيق التنمية الاقتصادية من خلال الثورة الرقمية التي تؤدي إلى نشوء أشكال جديدة تماماً من التفاعل الاجتماعي والاقتصادي وقيام مجتمعات جديدة. 
الثانية: زيادة قدرة الافراد على الاتصال وتقاسم المعلومات والمعارف ترفع من فرصية تحول العالم إلى مكان أكثر سلماً ورخاء لجميع سكانه، وهذا إذا ما كان جميع الافراد لهم إمكانيات المشاركة والاستفادة من هذه التكنولوجيا.

الثالثة: تمكن تكنولوجيا المعلومات بالإضافة إلى وسائل الإعلام التقليدية والحديثة، الافراد المهمشين والمعزولين من أن يدلوا بدلوهم في المجتمع العالهي، بغض النظر عن نوعهم أو مكان سكنهم، وهي تساعد على التسوية بين القوة وعلاقات صنع القرار على المستويين المحلي والدولي. وبوسعها تمكين الأفراد، والمجتمعات، والبلدان من تحسين مستوى حياتهم على نحو لم يكن ممكناً في السابق. ويمكنها

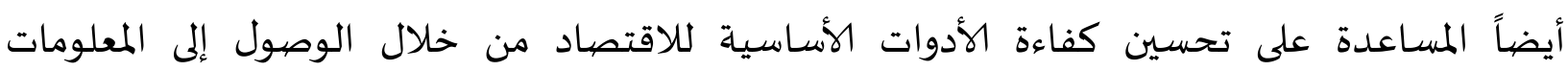
والشفافية.

الرابعة: تتميز تكنولوجيا المعلومات بالتلازمياة، وتعني إمكانية استقبال الرسالة في أي وقت مناسب المستخدم، فالمشاركين غير مطالبين باستخدام النظام في نفس الوقت، ففي البريد الإلكتروني مثلا نجد إلمديد

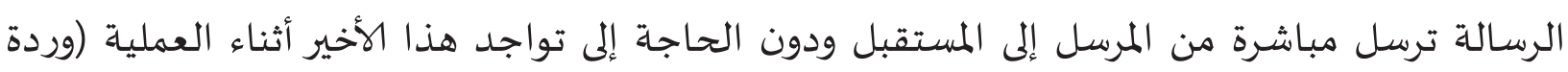
and وهيبة، 2013). وخلاصة لما سبق ان اهم خصائص تكنولوجيا المعلومات هي السرعة في معالجة البيانات الكترونيا وامكانية توصيلها الى جميع المستخدمين في جميع انحاء العالم بالوقت المناسب لاتخاذ القرارات.

\section{مُبْز مناقشَة}

علاقة تكنولوجيا المعلومات والأداء المنظمي

تكنولوجيا المعلومات اصبحت عاملا محفزا للتغيرات الرئيسة في الهيكل والعمليات وإدارة المنظمة،

وذلك ناتج من قدرتها على تحسين الانتاجياة، وتخفيض الكلف، تحسين اتخاذ القرارات فضلا عن تعزيز

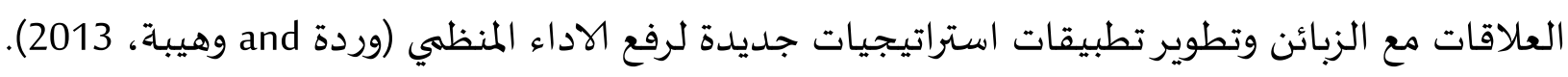

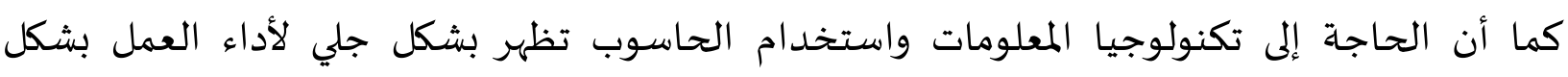

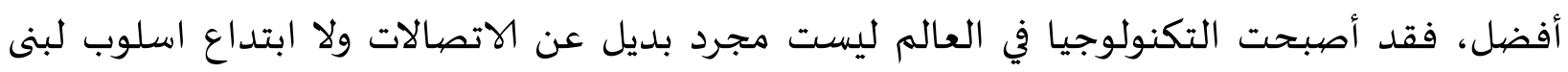
تحتية تتاح للعاملين انما تعد غاية لتحقيق مستويات عالية في الاداء اذ تساعد وتمكن المدراء من احداث تحسينات فائقة في اعمال المنظمة من خلال توفير المعلومات لاتخاذ قرارات فاعلة تدعم في تحقيق اداء 
منظمي فاعل (توربان et al. فالأداء يعد النتيجة النهائية لأي نشاط، ويشمل معرفة ما يجب 2000 أدائه، ومتى يجب أدائه؟ فضلا عن كيفية تقييماء؟ اذ عندما يتم استخدام تكنولوجيا المعلومات في التوصل لهذه النتائج وكيفية أداء الموظف لما هو مطلوب منه من خلال استخدامه لتلك التكنولوجيات

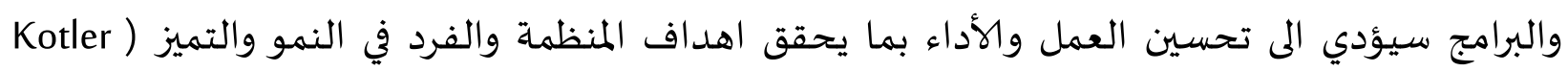
2000). كما تؤدي تكنولوجيا المعلومات دورا فاعلا بالنسبة للمنظمات ذات التوجه الاستراتيجي الساعي لهاتي لتعزيز القدرة التنافسية وذلك من خلال تعزيز كفاءة وفاعلية الأداء، اذ أن منظمات اليوم المتميزة والكفؤة لابد أن تتسم بامتلاكها تكنولوجيا المعلومات كي تتمكن من تحقيق الاداء العالي من خلال ما تقدمه من منتجات وخدمات متطورة فضلا عن تحسين عمليات الانتاج والتسويق وخفض الكلفة وتحسين الجودة في بيئة تتزايد فهها حدة المنافسة العالمية (Kotler 2000). فضلا عن دور تكنولوجيا المعلومات في تحديث وتطوير اداء المنظمات اذ تؤدي الى خلق أنواع جديدة من الوظائف ومجالات عمل ونشاطات متنوعة في بيئات العمل. اذ يمكن ملاحظة ذلك من خلال الآتي (اللوزي 2002):

1 2 ت تؤدي الى زيادة قنوات الاتصال الاداري بين مختلف الادارات. 3 توفر تحقيق رقابة فعالة في العمليات التشغيلية وتقليص حجم التنظيمات الادارية. 4

كما اكتسبت تكنولوجيا المعلومات أهميتها بسبب دورها الفاعل في تحسين الانتاجية وتحفيز العاملين والمدراء على بذل مجهودات فكرية مكثفة للإتيان بأشياء جديدة تؤدي الى الارتفاع والكفاءة في

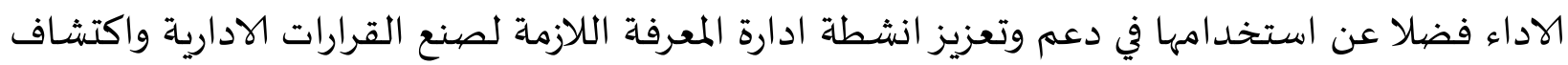
وتحليل المعرفة من خلال استخدام مكائن البحث وقواعد البيانات وايجاد الطرق الملائمة لتحديد الاهداف ووضع التكنولوجيا في مكانها المناسب (اللوزي 2002).

وتبرز علاقة تكنولوجيا المعلومات بالأداء المنظمي من خلال تأثيرها في الجانب المعنوي لدى العاملين باتجاه زيادة ولايهم وانتمائهم للمنظمة من خلال ما توفره من فرص للاطلاع على المعلومات بشكل سهل مما يسهم في تعزيز مشاركتهم في عملية صنع القرار (Liod and Rawlinson 1992). مما سبق القول نرى ان معظم المنظمات إذا ما ارادت ان تتميز بأدائها عن منافسيها لابد لها من استعمال تكنولوجيا المعلومات من خلال تطبيق عدد من الاجراءات وتدريب المستخدمين على هذه التكنولوجيا لضمان عملها بشكل صحيح وبما يحقق معايير الاداء الكفوء التي تسعى المنظمة الى تحقيقه. 
كما يبرز دور تكنولوجيا المعلومات في تحقيق مستويات عالية للأداء اذ تمكن الادارة العليا من احداث تحسينات وتطويرات فاعلة وكفؤة من خلال توفير المعلومات لاتخاذ قرارات بناءة تدعم رؤية ورسالة المنظمة مما يؤثر في الاهداف الاستراتيجية للمنظمة مع ضرورة توفير نوع من المرونة في استعمال التكنولوجيا وتطبيقها وفق اسلوب فاعل يؤدي الى اداء متميز للمنظمة اذ ان استعمال التكنولوجيا بشكل خاطئ قد يعيق عمل المنظمة ويؤدي بها الى الفشل والتدهور بدلا من أن يكون ذلك ميزة تنافسية ترفع من اداء المنظمة.

المطلب الأول: أدوار نظم المعلومات على مستوى المنظمة.

إن ضرورة المحاكاة للواقع الافتراضي، والحيوية والمرونة الهائلة التي يتمتع بها نظم المعلومات في توسيع مجال تطبيقه ليتجاوز الواقع الاقتصادي إلى غير، إذ صار من قبيل الحنكة في القيادة ليس فقط

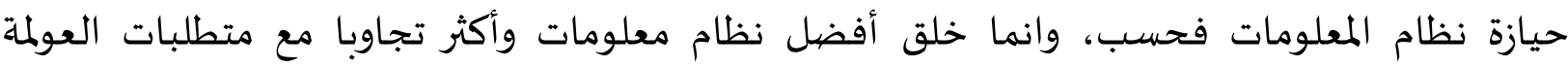

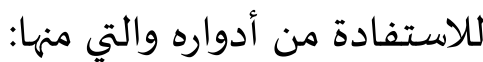
1 2 المشاركة في صياغة الرؤية الإستراتيجية لصالح متخذي القرار (هاشم 2006) من خلال توفر

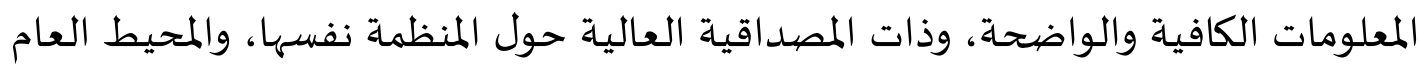
3

4 5 مما يقلل من الخسائر والتكاليف الباهظة، عن طريق التنبؤ بالبيئة الخارجية ورصد نقاط القوة

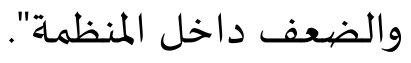

6 6 7 القيادة وكفاءة المستخدمين باتخاذ القرارات السليمة دون اللجوء للقيادة العليا، مما ينعكس إيجابا على السير الحسن للمؤسسة وتفعيل استغلال كافة مواردها. 8 "زيادة روح المشاركة والتعاون والمبادرة الذاتية والجماعياة، وروح الفريق المتألق"،(رايس 2005) وماله من أثرفي "تحقيق الاندماج البنيوي بين الأنشطة الجوهرية للرقابة ، والتقييم الاستراتيجي ومن ثمة تحسين الأداء الكلي للمنظمة" (سعد 2005). هنا يمكننا القول إن نظم المعلومات تساعد في الوصول إلى حالة من التفاعل الحي والفعلي، وهو ما يوفر المهارة الذاتية لكل عامل 
بغية التحكم في كل ما يطرأ على المؤسسـة، سواء من قريب أو بعيد، كما تخلق نوع من التأهب عند الطوارئ والتدخل بأسرع وقت ممكن بأنجح الحلول وأقلها ضررا.

$$
\text { نظم المعلومات سر نجاح كل منظمة }
$$

إذا كان الظاهر في نجاح أي منظمة،، هو قدرتها على اتخاذ القرارات المناسبة،، فإن السروالمفتاح

في ذلك هو التدفق للمعلومات المناسبة ذات المصداقية في الوقت المناسب، لذا فقد كان من الضروري زيادة فعالية وكفاءة نظم المعلومات كأهم خطة لتطوير أداء كل منظمة، وتعظيم قدرتها التنافسية لتحقيق أهدافها في ظل العولمة والثورة التكنولوجية التي نعيشها اليوم. "وبصفة عامة تعد نظم المعلومات تلك المجموعة المعقدة من الوظائف الحيوية بفضل العديد من الوسائل" (Klein and Ratier 2012) وتعتبر المعلومة اللبنة الأسساسية لدعم اتخاذ القرارات والتنسيق وكذا الرقابة داخل المنظمة.

\section{خلاصة}

تناولنا في بحثنا هذا تكنولوجيا المعلومات وأهميتها في دعم التطوير التنظيمي، ومن خلال الدراسـة التي اجريناها في هذا البحث تحصيّلنا على بعض الاستنتاجات التي تتلخص: 1) ينبغي أن نعتبر أن تكنولوجيا المعلومات أداةً ممكنة لإحداث التطوير والتحسين وتحقيق أهداف المؤسسـة 2) أصبح تأثير التكنولوجيات الجديدة عموماً وتكنولوجيا المعلومات خصهوصاً من المُسلَّمات في التنمية الاقتصادية والاجتماعية لإحداث ما أصبح يصطلح على تسميته بمجتمع المعلومات ومجتمع المعرفة؛ 3) أصبح امتلاك تكنولوجيا المعلومات واستثمارها من المعايير الهامة التي يقاس على أساسها تطوُّر البلدان وزيادة قُدرتها التنافسية؛ 4) تلعب تكنولوجيا المعلومات في إطار نظرية النظم دوراً أساسياً في بناء وتطوير وتحسين أداء نظم المعلومات في المؤسسة. تلعب تكنولوجيا المعلومات الآن الدور الأسـاسي في إعادة تشكيل المؤسسات، بحيث تفرض هذه التكنولوجيا وضرورات التعامل الإلكتروني في المؤسسات المعاصرة أهمية تطوير نموذجاً إداريّا يتميز بالانفتاح والمرونة والقُدرة على التكيُّف مع التطوُّرات التقنية.

\section{مراجع}

Daft, Richard L. 2000. Management. New York: The Dryden Press.

Klein, Tristan and Daniel Ratier. 2012. Rapport Sur L'impact Des TIC Sur Les Conditions

De Travail. France: Février.

Kotler, P. 2000. Marketing Manageme. New Jersey: PrenticeHall. 
Liod, C. and M. Rawlinson. 1992. New Technology and Human Resources Management. London.

Loudon , K, C. and P. Loudon , J. 2004. Management Information System. New Jersey: Prentice - Hill.

Slack , Nigel \& Chambens , S. \& Harland , C. \& Harrision , A. \& Johanston, R. 1998. Operation Management. London: Prentice Pub.

Turban, E., E. Miclean, and we Therbey J. 1999. Information Tecnology for Management, Making Connections for Strategic Advantege. New York: John wiley \& Sons. Inc.

اللوزي, موسى. 2002. التنمية الإدارية. الأردن: دار وائل. بركات, خالد مصطفى. 2005. “أثر استخدام تكنولوجيا المعلومات على الأداء المؤسسي للهيئات العامة العاملة في مجال المواصالات والنقل البري: دراسة تطبيقية على الهيئة القومية لسكك حديد مصر." كلية الاقتصاد والعلوم السياسية، جامعة القاهرة. توربان, افرايم ومكلين, افرايم ويثرب and,حميس. 2000. تقنية المعلومات في دعم إدارة الشركات. عمان: دار الرضيا للنشر.

حجيم, يوسف, والعبادي, هاشم فوزي and ,لطائي. 2005. ". إدارة الجودة الشاملة في التعليم الجامعي.” مجلة الغري للعلوم الاقتصيادية والإدرية 1(3).

رايس, مراد. 2005. “أثر تكنولوجية المعلومات على الموارد البشرية في المؤسسـة ـ دراسة حالة: مديرية الصيانة لسوناطراك بالأغواط'DML' تخصص إدارة أعمال." جامعة الجزائر. سعد, ياسين. 2005. تحليل وتصهيهم نظمر المعلومات. عمان: دار المناهج للنشروالتوزيع. صبري, هالة. 2002. تكنولوجيا المعلوهات ودورها في تعزيزمشاركة العاملين. عمان: ايار. منير, نوري ونعيمة and ربارك. 2005. "تكنولوجيا المعلومات والاتصال وأهميتها في اقتصاديات الدول العربية لمسايرة تحديات الاقتصاد العالمي الجديد -التوصيات والمتطلبات."جامعة حسيبة بن بوعلي الشلف.

هاشم, طارق نائل. 2006. "دور نظم المعلومات التسويقية في تحسين الأداء التسويقي للشركات المساهمة العامة الأردنية." جامعة عمان العربية للدراسـات العليا.

وردة, درب and قمودة وهيباة،. 2013. “استخدام تكنولوجيا المعلومات وتأثيرها على وظائف المؤسسـة دراسـة حالة بمؤسسة اتصالات الجزائروحدة ورقلة".

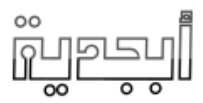

